\title{
Evaluation of the osteogenic differentiation of gingiva-derived stem cells grown on culture plates or in stem cell spheroids: Comparison of two- and three-dimensional cultures
}

\author{
SUNG-IL LEE, YOUNGKYUNG KO and JUN-BEOM PARK \\ Department of Periodontics, College of Medicine, The Catholic University of Korea, Seoul 06591, Republic of Korea
}

Received September 8, 2016; Accepted March 24, 2017

DOI: $10.3892 / \mathrm{etm} .2017 .4813$

\begin{abstract}
Three-dimensional cell culture systems provide a convenient in vitro model for the study of complex cell-cell and cell-matrix interactions in the absence of exogenous substrates. The current study aimed to evaluate the osteogenic differentiation potential of gingiva-derived stem cells cultured in two-dimensional or three-dimensional systems. To the best of our knowledge, the present study is the first to compare the growth of gingiva-derived stem cells in monolayer culture to a three-dimensional culture system with microwells. For three-dimensional culture, gingiva-derived stem cells were isolated and seeded into polydimethylsiloxane-based concave micromolds. Alkaline phosphatase activity and alizarin red $\mathrm{S}$ staining assays were then performed to evaluate osteogenesis and the degree of mineralization, respectively. Stem cell spheroids had a significantly increased level of alkaline phosphatase activity and mineralization compared with cells from the two-dimensional culture. In addition, an increase in mineralized deposits was observed with an increase in the loading cell number. The results of present study indicate that gingiva-derived stem cell spheroids exhibit an increased osteogenic potential compared with stem cells from two-dimensional culture. This highlights the potential of three-dimensional culture systems using gingiva-derived stem cells for regenerative medicine applications requiring stem cells with osteogenic potential.
\end{abstract}

\section{Introduction}

Mesenchymal stem cells are a major cell source for regenerative medicine due to their multilineage potential (1). Stem cells have been isolated and characterized from human gingiva, demonstrating multipotency with a high rate of proliferation

Correspondence to: Dr Jun-Beom Park, Department of Periodontics, College of Medicine, The Catholic University of Korea, 222 Banpo-daero, Seoul 06591, Republic of Korea E-mail: jbassoonis@yahoo.co.kr

Key words: cell culture techniques, cell differentiation, cellular spheroids, stem cell research and exhibiting characteristics of mesenchymal stem cells (2). Gingiva-derived stem cells have been applied for tissue regeneration, including bone and periodontal regeneration (3-5). Systemically transplanted enhanced green fluorescent protein (eGFP)-labeled gingiva-derived stem cells were administered via the tail vein, where the stem cells concentrated at the mandibular defect and promoted bone regeneration (4). In another study, eGFP-labeled gingiva-derived stem cells were seeded onto type I collagen gel and implanted into mandibular defects and calvarial defects in Sprague Dawley rats (6). Previous reports have indicated that gingiva-derived stem cells significantly enhance the regeneration of the damaged periodontal tissue, including the alveolar bone, cementum and functional periodontal ligament (3). In addition, it has been demonstrated that gingiva-derived stem cells and bone marrow mesenchymal stromal cells have a similar osteogenic capacity (5).

While traditional cell culture methods have relied on growing cells as monolayers, three-dimensional culture systems may provide a convenient in vitro model for the study of complex cell-cell and cell-matrix interactions in the absence of exogenous substrates (7). Furthermore, mesenchymal stem cells are typically cultured on a two-dimensional substrate in vitro, and cells may lose their stemness as a result of prolonged two-dimensional culture $(8,9)$. The present study aimed to evaluate the osteogenic differentiation of gingiva-derived stem cells using two-dimensional and three-dimensional culture systems. To the best of our knowledge, the current study is the first to evaluate and compare the use of two-dimension and microwell-based three-dimensional culture systems for gingiva-derived stem cells.

\section{Materials and methods}

Isolation and culture of gingiva-derived stem cells. Gingiva-derived stem cells were obtained using a previously described method (2). The present study was reviewed and approved by the Institutional Review Board of Seoul St. Mary's Hospital (Seoul, Korea; approval no. KC11SISI0348). Gingival tissues were collected under local anesthesia using a surgical scalpel blade from a 14 year-old male patient who was referred to the Department of Periodontics at Seoul St Mary's Hospital for a crown lengthening procedure in August 2013. Written informed consent was obtained from the patient's legal guardian. Gingival tissues were de-epithelialized, minced 
and digested in a $\alpha$-modified Minimal Essential Medium ( $\alpha$-MEM; Gibco; Thermo Fisher Scientific, Inc., Waltham, MA, USA) containing collagenase IV (2 mg/ml; Sigma-Aldrich; Merck KGaA, Darmstadt, Germany) and dispase $(1 \mathrm{mg} / \mathrm{ml}$; Sigma-Aldrich; Merck KGaA). Non-adherent cells were washed away with PBS (Welgene, Gyeongsan, South Korea) twice and fresh medium was added to the culture in a $37^{\circ} \mathrm{C}$ humidified incubator containing $5 \% \mathrm{CO}_{2}$.

Formation of stem cell spheroids. Stem cell spheroids were formed in $600 \mu \mathrm{m}$ diameter silicon elastomer (polydimethylsiloxan)-based concave microwells (StemFIT 3D ${ }^{\circledR}$; MicroFIT, Seongnam, Korea) in a $37^{\circ} \mathrm{C}$ humidified incubator containing $5 \% \mathrm{CO}_{2}$. Then, $8 \times 10^{5}$ stem cells $/ 100$ concave micromolds were seeded and cultured in $\alpha$-MEM or osteogenic induction media at $37^{\circ} \mathrm{C}$. Spheroid formations were observed and imaged under an inverted fluorescence microscope (CKX41SF, Olympus Corporation, Tokyo, Japan).

Alkaline phosphatase activity assay. Cell cultures were grown at $8 \times 10^{3}$ stem cells $/ \mathrm{mm}$ in a $37^{\circ} \mathrm{C}$ humidified incubator containing $5 \% \mathrm{CO}_{2}$ (Fig. 1) with $\alpha$-MEM or osteogenic induction media (StemPro ${ }^{\circledR}$ Osteogenesis Differentiation kit; Gibco; Thermo Fisher Scientific, Inc.) were harvested on days 1, 3 and 7. Cells were detached using trypsin (Gibco; Thermo Fisher Scientific, Inc.) and washed twice with PBS for $1 \mathrm{~min}$. Alkaline phosphatase activity assays were performed using the Alkaline Phosphatase Activity Colorimetric assay kit (cat no. K412-500; BioVision, Inc., Milpitas, CA, USA) according to the manufacturer's protocol. The cells were resuspended with assay buffer, sonicated at $70-80 \%$ intensity for $1 \mathrm{~min}$ at $4^{\circ} \mathrm{C}$ and then centrifuged at $13,000 \mathrm{x}$ g for $3 \mathrm{~min}$ at $4^{\circ} \mathrm{C}$ to remove insoluble material. The supernatant was mixed with p-nitrophenylphosphate substrate (provided in the kit) and incubated at $25^{\circ} \mathrm{C}$ for $60 \mathrm{~min}$. The optical density of the resultant p-nitrophenol at $405 \mathrm{~nm}$ was determined spectrophotometrically.

Stem-cell spheroids formed in the concave micromolds were moved to culture plates on day 3. The spheroids were grown with $\alpha$-MEM or osteogenic induction media in a $37^{\circ} \mathrm{C}$ humidified incubator containing $5 \% \mathrm{CO}_{2}$ (Fig. 1) and harvested on days 1,3 and 7. Alkaline phosphatase activity assays were performed as described above on the spheroids.

Alizarin red Staining for mineralization. Gingiva-derived stem cell groups of $4 \times 10^{5}$ (group 1) or $8 \times 10^{5}$ (group 2) cells were seeded on a $100 \mathrm{~mm}$ diameter culture plate and grown with $\alpha$-MEM or osteogenic induction media. Stem-cell spheroids formed in the concave micromolds at $4 \times 10^{5}$ (group 1) or $8 \times 10^{5}$ (group 2) cells/100 concave micromolds were moved to the culture plate at day 3 and grown with $\alpha$-MEM or osteogenic induction media. On day 6, cells and spheroids were washed twice with PBS for $1 \mathrm{~min}$, fixed with $70 \%$ ethanol at room temperature for $15 \mathrm{~min}$ and rinsed twice with deionized water. The two- and three-dimensional cultures were stained with alizarin red $\mathrm{S}$ for $30 \mathrm{~min}$ at room temperature. To remove non-specifically bound dye, the cultures were washed 3 times for 2 min with deionized water and then with PBS for $15 \mathrm{~min}$ at room temperature. Bound dye was solubilized in $10 \mathrm{mM}$ sodium phosphate containing $10 \%$ cetylpyridinium chloride (Sigma-Aldrich; Merck KGaA) and quantified spectrophotometrically at a wavelength of $560 \mathrm{~nm}$.

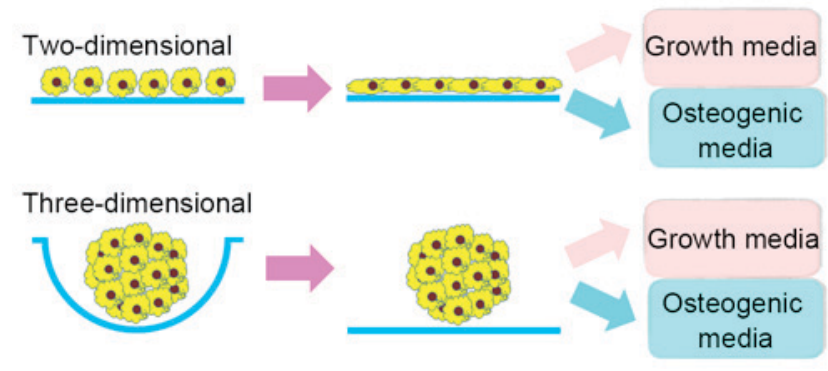

Figure 1. Schematic illustration of the method used in the present study, comparing the growth of gingival-derived stem cells using two- and three-dimensional culture techniques.

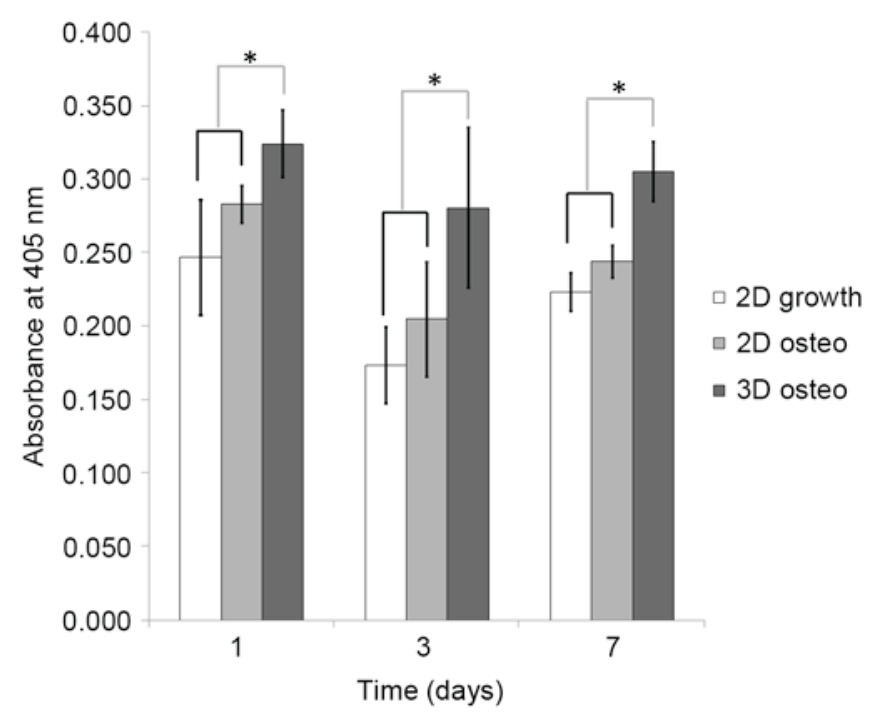

Figure 2. Alkaline phosphatase activity is increased in gingival-derived stem cells grown in three-dimensional culture. ${ }^{*} \mathrm{P}<0.05$. 2D growth, two-dimensional growth in normal media; $2 \mathrm{D}$ osteo, two-dimensional growth in osteogenic media; 3D osteo, three-dimensional growth in osteogenic media.

Statistical analysis. Data are presented as the mean \pm standard deviation. A Shapiro-Wilk test for normality was performed, and a one-way analysis of variance with a post hoc Tukey's range test was performed to determine the statistical significance of differences between groups. SPSS software (version 12; SPSS Inc., Chicago, IL, USA) was used for all statistical tests. $\mathrm{P}<0.05$ was determined to indicate a statistically significant difference.

\section{Results}

Alkaline phosphatase activity assays. The results of the alkaline phosphatase activity assays are presented in Fig. 2. All the comparisons are made between the three-dimensional osteogenic group and the two-dimensional growth and two-dimensional osteogenic groups. The absorbance at $405 \mathrm{~nm}$ on day 1 was significantly lower for the two-dimensional compared with the three-dimensional osteogenic differentiation group $(0.283 \pm 0.013$ and $0.324 \pm 0.023 \mathrm{~nm}$, respectively; $\mathrm{P}<0.05)$. The absorbance values on day 3 were significantly lower for the two-dimensional compared with the three-dimensional osteogenic differentiation group $(0.205 \pm 0.039$ and $0.281 \pm 0.054 \mathrm{~nm}$, respectively; $\mathrm{P}<0.05)$. Similarly, the 

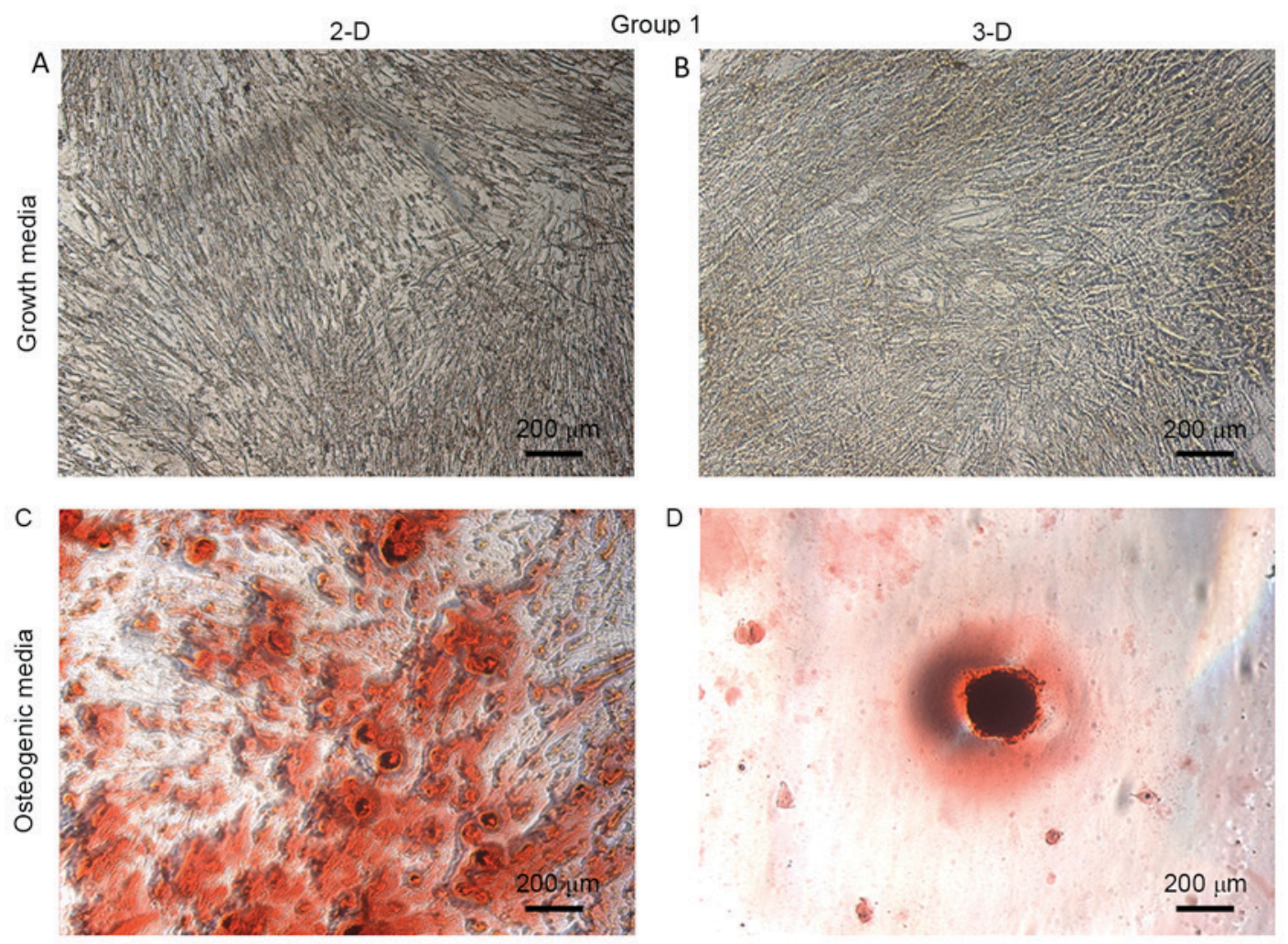

2-D

Group 2

3-D
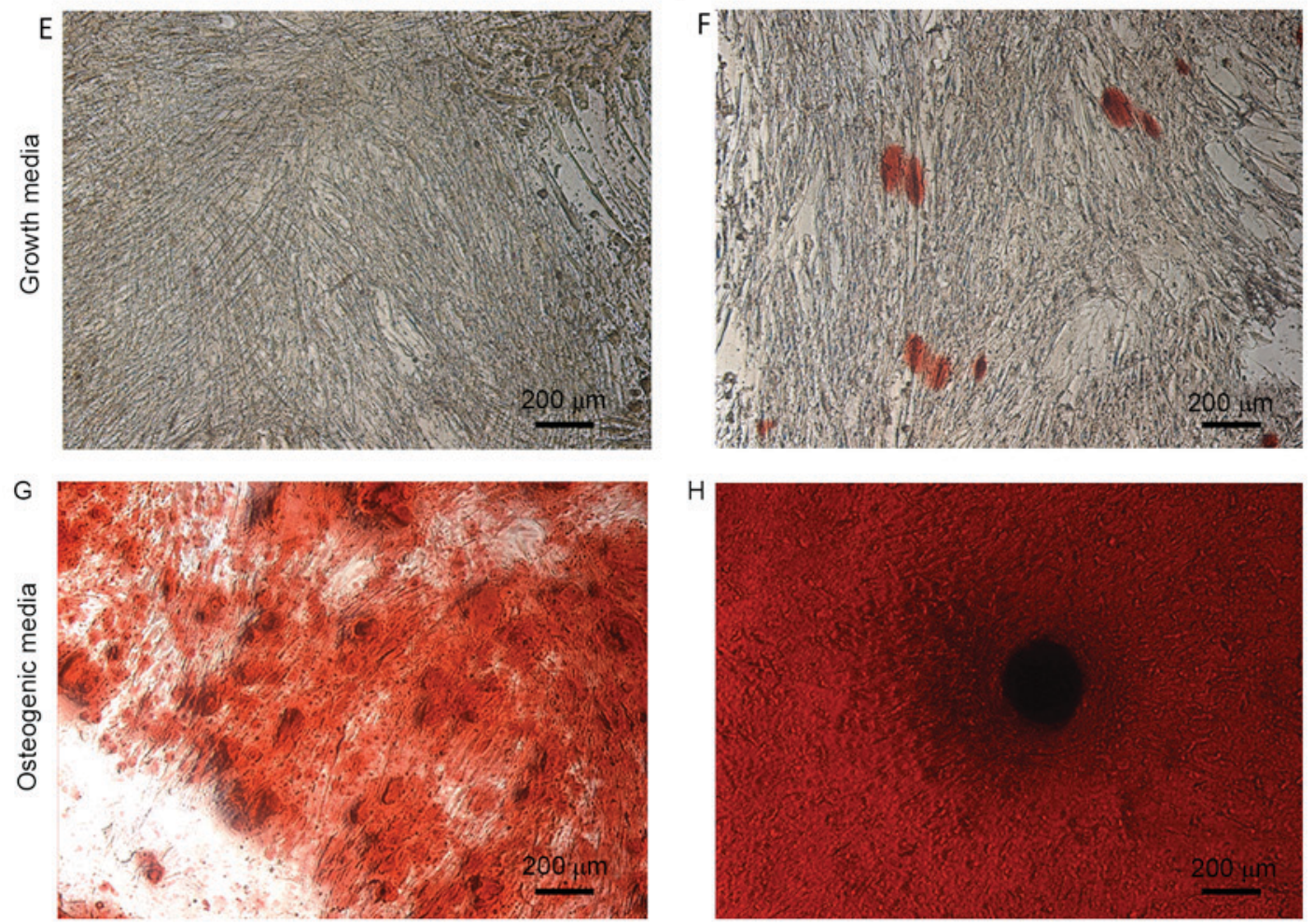

Figure 3. Mineralization is increased in gingival-derived stem cells grown in three-dimensional culture. Results of alizarin red S staining of gingiva-derived stem cells on day 6 (magnification, x100). Growth of cells in group $1\left(4 \times 10^{5}\right.$ cells) following (A) two-dimensional and (B) three-dimensional growth in normal media, and (C) two-dimensional and (D) three dimensional growth in osteogenic induction media. Growth of cells in group 2 (8x10 $0^{5}$ cells) following (E) two-dimensional and (F) three-dimensional growth in normal media, and (G) two-dimensional and (H) three dimensional in osteogenic induction media.

absorbance values on day 7 were significantly lower for the two-dimensional compared with the three-dimensional osteogenic differentiation group $(0.244 \pm 0.011$ and $0.305 \pm 0.020 \mathrm{~nm}$, respectively; $\mathrm{P}<0.05$ ).
Mineralization assay. Minimal mineralized extracellular deposits were observed following the alizarin red S staining of two-dimensional and three-dimensional growth media groups (Fig. 3A and B). An increase in the number of mineralized 


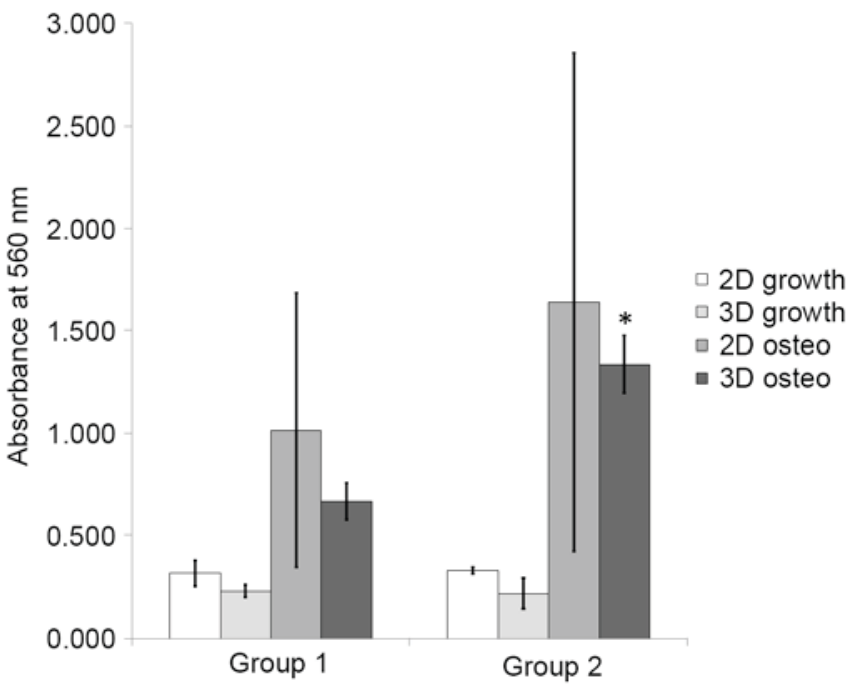

Figure 4. Quantification of the results of the mineralization assay on day 6 . ${ }^{*} \mathrm{P}<0.05$ vs. group $13 \mathrm{D}$ osteo $\left(4 \times 10^{5}\right.$ cells $)$. $2 \mathrm{D}$ growth, two-dimensional growth in normal media; 3D growth, three-dimensional growth in normal media; 2D osteo, two-dimensional growth in osteogenic media; 3D osteo, three-dimensional growth in osteogenic media.

deposits was revealed in the osteogenic media groups, and more intense staining was observed in group 2 compared with group 1 (Fig. 3). The quantitative results regarding bound dye are presented in Fig. 4. Quantitative absorbance values for group 1 on day 6 were $0.317 \pm 0.062,0.234 \pm 0.030$, $1.016 \pm 0.667$ and $0.670 \pm 0.091 \mathrm{~nm}$ for two-dimensional growth, three-dimensional growth, two-dimensional osteogenic and three-dimensional osteogenic groups, respectively $(\mathrm{P}=0.076)$. The absorbance values for group 2 were $0.330 \pm 0.018$, $0.221 \pm 0.073,1.639 \pm 1.216$ and $1.339 \pm 0.139 \mathrm{~nm}$ for two-dimensional growth, three-dimensional growth, two-dimensional osteogenic and three-dimensional osteogenic groups, respectively $(\mathrm{P}=0.050)$.

\section{Discussion}

The present study compared the effects of the osteogenic differentiation of gingiva-derived stem cells using two-dimensional and three-dimensional culture systems and demonstrated that gingiva-derived stem cell spheroids exhibited an increased osteogenic potential from alkaline phosphatase activity assays and alizarin red $\mathrm{S}$ staining when compared with stem cells cultured using the two-dimensional culture method.

Various methods may be used to form cell spheroids (10-13). A previous study reported that multicellular aggregates were formed using the hanging droplet technique (10). In addition, the formation of stem cell spheroids may be induced using a rotating platform (11). Microwell culture systems have previously been applied to generate homogenous stem cell colonies of defined sizes and shapes (12). A previous study used polyethylene glycol microwells to produce easily retrievable cell aggregates with high viability and controlled sizes and shapes defined by the geometry of the microwells (13). The present study used polydimethylsiloxane-based concave micromolds to form stem cell spheroids.
It has been suggested that three-dimensional culture systems may be applied in regenerative medicine strategies (7). Three-dimensional culture systems are reported to have a number of advantages (14). Three-dimensional cultures allow the long-term maintenance of stem cells without the routine passaging and manipulation necessary for traditional two-dimensional cell propagation $(15,16)$. The available surface area for the propagation of cells is limited in two-dimensional systems, whereas three-dimensional systems have been developed for the expansion of stem cells as spheres (11). Stem cell spheroids exhibit greater resistance to apoptosis (17). In addition, stem cell spheroids kept in the three-dimensional system secrete considerable amounts of vascular endothelial growth factor $\mathrm{A}$, which was undetected in two-dimensional cultures (18).

Mesenchymal stem cells are typically cultured on a two-dimensional substrate in vitro and cells may lose their multipotentiality as a result of prolonged two-dimensional culture $(8,9)$. The three-dimensional spheroid culture system has demonstrated efficient multipotency, including osteogenic differentiation, compared with two-dimensional culture $(1,11,19)$. Similarly, the three-dimensional culturing of human adipose-derived stem cells as multicellular aggregates caused elevated levels of osteogenic differentiation and increased matrix mineralization when compared with analogous cells in monolayer culture (10). The results of the present study demonstrated that three-dimensional cultures of gingiva-derived stem cells exhibited a statistically significant increase in alkaline phosphatase activity compared with those in two-dimensional culture. A previous study revealed that three-dimensional stem cell spheres exhibited 10-fold higher mineralization compared with the two-dimensional control culture (20). Similarly, the expression of alkaline phosphatase was reported to be higher in three-dimensional scaffold-free stem-cell sheet-derived pellets compared with the cell sheets (10). Another study demonstrated that three-dimensional stem cell spheres possessed 4-fold greater alkaline phosphatase activity compared with the two-dimensional control culture (20).

Three-dimensional culture may be broadly categorized into scaffold-free or scaffold-based culture systems, with scaffolds made from either natural or synthetic materials (14). Biomaterial-supported culture methods, allowing for the directed three-dimensional differentiation of stem cells, are an alternative to canonical two-dimensional cell cultures (21). A polymer matrix can be used for the three-dimensional culture to promote of osteogenesis (22). Another study used a collagen/hydroxyapatite scaffold for stem cell culture to promote proliferation and maintain differentiation capacity (9). Encapsulation of stem cells within alginate beads may support and enhance differentiation compared with two-dimensional cultures (21). In addition, a previous study indicated that the structure of the substrate used exhibits potent effects on cell function compared with the substrate composition when nanofiber scaffolds were compared with flat films, yielding marked differences in gene expression (23).

Human mesenchymal stem cells have potential applications in stem cell therapy due to their wide availability, immunomodulatory properties and multipotent nature, which allows them to differentiate into chondrocytes, osteocytes 
and adipocytes (21). It has been suggested that mesenchymal stem cells derived from gingival tissue may be a novel cell source for stem cell therapy in bone reconstruction (6). The results of a previous study suggested that a three-dimensional spheroid culture approach optimized the stem cell properties of enhanced multipotency and therapeutic effects of chemokine secretion from human gingiva-derived mesenchymal stem cells (24). In addition, the production of uniform spheroids allows for improved handling and manipulation of the cells (10).

In conclusion, the current study demonstrated that gingiva-derived stem cell spheroids exhibited increased osteogenic potential when compared with stem cells cultured using the two-dimensional culture method. This highlights the potential of three-dimensional culture systems using gingiva-derived stem cells for regenerative medicine applications requiring osteogenic potential.

\section{Acknowledgements}

The present study was supported by the Basic Science Research Program through the National Research Foundation of Korea, which is funded by the Ministry of Science, Information and Communication Technology \& Future Planning (Daejeon, South Korea; grant no. NRF-2014R1A1A1003106).

\section{References}

1. Yamaguchi Y, Ohno J, Sato A, Kido H and Fukushima T: Mesenchymal stem cell spheroids exhibit enhanced in-vitro and in-vivo osteoregenerative potential. BMC Biotechnol 14: 105, 2014.

2. Jin SH, Lee JE, Yun JH, Kim I, Ko Y and Park JB: Isolation and characterization of human mesenchymal stem cells from gingival connective tissue. J Periodontal Res 50: 461-467, 2015.

3. Yu X, Ge S, Chen S, Xu Q, Zhang J, Guo H and Yang P: Human gingiva-derived mesenchymal stromal cells contribute to periodontal regeneration in beagle dogs. Cells Tissues Organs 198: 428-437, 2013.

4. Xu QC, Wang ZG, Ji QX, Xu XY, Yuan CQ, Deng J and Yang PS: Systemically transplanted human gingiva-derived mesenchyma stem cells contributing to bone tissue regeneration. Int J Clin Exp Pathol 7: 4922-4929, 2014

5. Zorin VL, Komlev VS, Zorina AI, Khromova NV, Solovieva EV, Fedotov AY, Eremin II and Kopnin PB: Octacalcium phosphate ceramics combined with gingiva-derived stromal cells for engineered functional bone grafts. Biomed Mater 9: 055005, 2014.

6. Wang F, Yu M, Yan X, Wen Y, Zeng Q, Yue W, Yang P and Pei X: Gingiva-derived mesenchymal stem cell-mediated therapeutic approach for bone tissue regeneration. Stem Cells Dev 20 2093-2102, 2011.

7. Baraniak PR and McDevitt TC: Scaffold-free culture of mesenchymal stem cell spheroids in suspension preserves multilineage potential. Cell Tissue Res 347: 701-711, 2012.

8. Han S, Zhao Y, Xiao Z, Han J, Chen B, Chen L and Dai J: The three-dimensional collagen scaffold improves the stemness of rat bone marrow mesenchymal stem cells. J Genet Genomics 39: 633-641, 2012.

9. Antebi B, Zhang Z, Wang Y, Lu Z, Chen XD and Ling J: Stromal-cell-derived extracellular matrix promotes the proliferation and retains the osteogenic differentiation capacity of mesenchymal stem cells on three-dimensional scaffolds. Tissue Eng Part C Methods 21: 171-181, 2015.
10. Shen FH, Werner BC, Liang H, Shang H, Yang N, Li X, Shimer AL, Balian G and Katz AJ: Implications of adipose-derived stromal cells in a 3D culture system for osteogenic differentiation: An in vitro and in vivo investigation. Spine J 13: 32-43, 2013.

11. Cha HM, Kim SM, Choi YS and Kim DI: Scaffold-free three-dimensional culture systems for mass production of periosteum-derived progenitor cells. J Biosci Bioeng 120: 218-222, 2015.

12. Hsiao C and Palecek SP: Microwell regulation of pluripotent stem cell self-renewal and differentiation. Bionanoscience 2 : 266-276, 2012.

13. Karp JM, Yeh J, Eng G, Fukuda J, Blumling J, Suh KY, Cheng J, Mahdavi A, Borenstein J, Langer R and Khademhosseini A: Controlling size, shape and homogeneity of embryoid bodies using poly (ethylene glycol) microwells. Lab Chip 7: 786-794, 2007.

14. Knight E and Przyborski S: Advances in 3D cell culture technologies enabling tissue-like structures to be created in vitro. J Anat 227: 746-756, 2015.

15. Seymour T, Twigger AJ and Kakulas F: Pluripotency genes and their functions in the normal and aberrant breast and brain. Int $\mathrm{J}$ Mol Sci 16: 27288-27301, 2015.

16. Wei J, Han J, Zhao Y, Cui Y, Wang B, Xiao Z, Chen B and Dai J: The importance of three-dimensional scaffold structure on stemness maintenance of mouse embryonic stem cells. Biomaterials 35: 7724-7733, 2014.

17. Murphy KC, Fang SY and Leach JK: Human mesenchymal stem cell spheroids in fibrin hydrogels exhibit improved cell survival and potential for bone healing. Cell Tissue Res 357: 91-99, 2014.

18. Santos JM, Camoes SP, Filipe E, Cipriano M, Barcia RN, Filipe M, Teixeira M, Simões S, Gaspar M, Mosqueira D, et al: Three-dimensional spheroid cell culture of umbilical cord tissue-derived mesenchymal stromal cells leads to enhanced paracrine induction of wound healing. Stem Cell Res Ther 6: 90, 2015.

19. Jing Y and Jian-Xiong Y: 3-D spheroid culture of bone marrow mesenchymal stem cell of rhesus monkey with improved multi-differentiation potential to epithelial progenitors and neuron in vitro. Clin Experiment Ophthalmol 39: 808-819, 2011.

20. Singhatanadgit W and Varodomrujiranon M: Osteogenic potency of a 3-dimensional scaffold-free bonelike sphere of periodontal ligament stem cells in vitro. Oral Surg Oral Med Oral Pathol Oral Radiol 116: e465-e472, 2013.

21. Bozza A, Coates EE, Incitti T, Ferlin KM, Messina A, Menna E, Bozzi Y, Fisher JP and Casarosa S: Neural differentiation of pluripotent cells in 3D alginate-based cultures. Biomaterials 35: 4636-4645, 2014.

22. Guerrero J, Oliveira H, Catros S, Siadous R, Derkaoui SM, Bareille R, Letourneur D and Amédée J: The use of total human bone marrow fraction in a direct three-dimensional expansion approach for bone tissue engineering applications: Focus on angiogenesis and osteogenesis. Tissue Eng Part A 21: 861-874, 2015.

23. Baker BA, Pine PS, Chatterjee K, Kumar G, Lin NJ, McDaniel JH, Salit ML and Simon CG Jr: Ontology analysis of global gene expression differences of human bone marrow stromal cells cultured on 3D scaffolds or 2D films. Biomaterials 35: 6716-6726, 2014.

24. Zhang Q, Nguyen AL, Shi S, Hill C, Wilder-Smith P, Krasieva TB and Le AD: Three-dimensional spheroid culture of human gingiva-derived mesenchymal stem cells enhances mitigation of chemotherapy-induced oral mucositis. Stem Cells Dev 21: 937-947, 2012. 